\title{
Chimeric Antigen Receptor-Natural Killer Cells: The Future of Cancer Immunotherapy
}

\author{
Sana Habib, MBBS, ${ }^{1}$ Syed M. Tariq, MBBS, ${ }^{2}$ Moeez Tariq, MBBS ${ }^{1}$ \\ ${ }^{1}$ Department of Medicine, Dow University of Health Sciences, Karachi, Pakistan ${ }^{2}$ Department of Medicine, Jinnah Sindh Medical University, \\ Karachi City, Sindh, Pakistan
}

\section{TO THE EDITOR}

A new era of cancer immunotherapy has begun. Chimeric antigen receptor $T$ (CAR-T) cells, with $T$ cells genetically engineered to recognize CD-19 antigens of $B$ cells, have been confirmed to successfully treat relapsed and refractory B cell malignancies in various clinical trials. ${ }^{1}$ However, this treatment modality has several drawbacks. Side effects such as cytokine toxicity, tumor lysis syndrome, effects on healthy tissues, B cell aplasia, and genotoxicity may be fatal, aside from the neoplasm itself. ${ }^{2}$

CAR technology is being applied to other immune cells such as natural killer (NK) cells. NK cells are defined as CD56+ and CD3- cells and are subdivided into cytotoxic and immunoregulatory. They are of great clinical interest because they contribute to the graft-vs-leukemia/graft-vstumor effect but are not responsible for graft-vs-host disease. NK cells can be generated from various sources such as umbilical cord blood, bone marrow, human embryonic stem cells, and induced pluripotent stem cells. However, tumors can escape the cytotoxicity of NK cells when they are directed against NKG2D ligands MICA and MICB (major histocompatibility complex class I chain-related protein A/B). ${ }^{3}$ Henceforth, preclinical research has been reported for CAR-modified primary human NK cells redirected against CD19, CD20, CD244, and HER2, as well as CARexpressing NK-92 cells targeted to a wider range of cancer antigens. ${ }^{4}$

Primary NK cells engineered to express CARs have potential benefits compared to CAR-T cells. NK cells have spontaneous cytotoxic activity and can generate target cell death independent of tumor antigen, while T lymphocytes only kill their targets by a CAR-specific mechanism. Therefore, in the setting of antigen downregulation by tumor cells attempting to escape immune detection, NK cells would still be effective against tumor cells. In addition, primary human NK cells produce cytokines, such as interferon gamma, interleukin 3 , and granulocyte-macrophage colony-stimulating factor, that differ from the proinflammatory cytokines produced by $T$ cells that are responsible for the onset of cytokine release syndrome. Individual NK cells can survive after contacting and killing multiple target cells, possibly reducing the number of cells that need to be adoptively transferred (ie, the ex vivo stimulation and expansion of autologous or allogeneic lymphocytes, followed by reinfusion of the expanded lymphocyte population into the patient, in contrast to T cells). Furthermore, whereas the long-term persistence of CAR-T cells may maintain on-target, off-tumor toxicity such as the B cell aplasia seen with anti-CD19 CAR-T cells, mature NK cells are short lived and are expected to disappear after facilitating their anticancer effects. Therefore, NK cell lines are appealing for CAR cell therapy. ${ }^{5}$ The continued genetic manipulation of NK cells into antigen-specific effector cells could lead to improved responses of unmodified cells; however, key obstacles must be overcome if NK cellbased immunotherapies are to meet their perceived potential. These obstacles include the inability to traffic into solid tumor sites and avoid tumor immunosuppression and the resistance to current gene transfection techniques; culture and expansion techniques that promote the reproducible growth and expansion of NK cells for therapy must also be developed. ${ }^{6}$ The inability for CAR-NK cells to reproduce in vivo would lead to the need for an increased frequency and quantity of the product to be transferred, leading to a drain on resources and patient morale. Nevertheless, given the recent advances and rapid developments in clinically compliant techniques to expand and genetically manipulate NK cells, CAR-NK cell-based immunotherapy likely constitutes a promising prospect for cancer therapy.

Henceforth, NK cells could represent a method of immunotherapy that, unlike $\mathrm{T}$ cells, could be more readily fine-tuned to prevent treatment-associated toxicity and immune-mediated adverse events. More clinical trials are needed for the benefits of CAR-NK cells to be confirmed, but preclinical data have shown promising results. As relapsed and refractory $B$ cell malignancies continue to have poor prognosis despite newer treatment regimens, CAR-NK cell line therapy may provide a favorable treatment alternative. ${ }^{7}$

\section{REFERENCES}

1. Chen Y, Liu D. Chimeric antigen receptor (CAR)-directed adoptive immunotherapy: a new era in targeted cancer therapy. Stem Cell Investig. 2014 Jan 18;1:2. doi: 10.3978/j.issn.2306-9759.2013.12.01.

2. Kalaitsidou M, Kueberuwa G, Schütt A, Gilham DE. CAR T-cell therapy: toxicity and the relevance of preclinical models. Immunotherapy. 2015;7(5):487-497. doi: 10.2217/imt.14.123.

3. Holdenrieder S, Eichhorn P, Beuers $U$, et al. Soluble NKG2D ligands in hepatic autoimmune diseases and in benign diseases involved in marker metabolism. Anticancer Res. 2007 Jul-Aug;27(4A):2041-2045.

4. Glienke W, Esser R, Priesner C, et al. Advantages and applications of CAR-expressing natural killer cells. Front Pharmacol. 2015 Feb 12;6:21. doi: 10.3389/fphar.2015.00021. 
5. Bollino D, Webb TJ. Chimeric antigen receptor-engineered natural killer and natural killer T cells for cancer immunotherapy. Transl Res. 2017 Sep;187:32-43. doi: 10.1016/j.trsl.2017.06.003.

6. Grossenbacher SK, Aguilar EG, Murphy WJ. Leveraging natural killer cells for cancer immunotherapy. Immunotherapy. 2017 May;9(6):487-497. doi: 10.2217/imt-2017-0013.
7. Schuster SJ, Svoboda J, Chong EA, et al. Chimeric antigen receptor T cells in refractory B-cell lymphomas. N Engl J Med. 2017 Dec 28;377(26):2545-2554. doi: 10.1056/NEJMoa1708566. 